Article

\title{
Genome Sequence of Dickeya solani, a New soft Rot Pathogen of Potato, Suggests its Emergence May Be Related to a Novel Combination of Non-Ribosomal Peptide/Polyketide Synthetase Clusters
}

\section{Linda Garlant ${ }^{1}$, Patrik Koskinen ${ }^{2}$, Leo Rouhiainen ${ }^{3}$, Pia Laine ${ }^{4}$, Lars Paulin ${ }^{4}$, Petri Auvinen ${ }^{4}$, Liisa Holm ${ }^{2,4}$ and Minna Pirhonen ${ }^{1, *}$}

1 Department of Agricultural Sciences, P.O. Box 27, Latokartanonkaari 7, University of Helsinki, 00014, Finland; E-Mail: linda.garlant@ helsinki.fi

2 Department of Biosciences, Division of Genetics, P.O. Box 56, Viikinkaari 9, University of Helsinki, 00014, Finland; E-Mails: patrik.koskinen@ helsinki.fi (P.K.); liisa.holm@helsinki.fi (L.H.)

3 Department of Food and Environmental Sciences, Division of Microbiology, P.O. Box 56, Viikki Biocenter, Viikinkaari 9, University of Helsinki, 00014, Finland;

E-Mail: leo.rouhiainen@helsinki.fi

4 Institute of Biotechnology, P.O.Box 56, Viikinkaari, University of Helsinki, 00014, Finland; E-Mails: pia.k.laine@helsinki.fi (P.L.); lars.paulin@helsinki.fi (L.P.); petri.auvinen@helsinki.fi (P.A.)

* Author to whom correspondence should be addressed; E-Mail: minna.pirhonen@helsinki.fi; Tel.: +358-9-191-59621; Fax: +358-9-191-58727.

Received: 25 September 2013; in revised form: 28 October 2013 / Accepted: 14 November 2013 / Published: 6 December 2013

\begin{abstract}
Soft rot Enterobacteria in the genera Pectobacterium and Dickeya cause rotting of many crop plants. A new Dickeya isolate has been suggested to form a separate species, given the name Dickeya solani. This bacterium is spreading fast and replacing the closely related, but less virulent, potato pathogens. The genome of $D$. solani isolate D s0432-1 shows highest similarity at the nucleotide level and in synteny to $D$. dadantii strain 3937, but it also contains three large polyketide/fatty acid/non-ribosomal peptide synthetase clusters that are not present in $D$. dadantii 3937. These gene clusters may be involved in the production of toxic secondary metabolites, such as oocydin and zeamine. Furthermore, the D. solani genome harbors several specific genes that are not present in other Dickeya and Pectobacterium species and that may confer advantages for adaptation
\end{abstract}


to new environments. In conclusion, the fast spreading of $D$. solani may be related to the acquisition of new properties that affect its interaction with plants and other microbes in the potato ecosystem.

Keywords: Dickeya solani; genome comparison; NRPS; PKS

\section{Introduction}

The genus Dickeya contains several species of Gram-negative, opportunistic, pectinolytic plant pathogenic bacteria. This genus derives from the reclassification of Pectobacterium chrysanthemi (synonym Erwinia chrysanthemi) [1] into six new genomic species: D. chrysanthemi, D. paradisiaca, D. dadantii, D. dianthicola, D. dieffenbachiae and D. zeae [2]. Recently, D. dieffenbachiae was reclassified as a subspecies of $D$. dadantii. Dickeya species are broad host-range phytopathogens that cause soft rot in numerous plant species, including potato [3]. Most Dickeya strains are considered to be adapted to high temperatures and are thus common in plants in warm and tropical climates [4]. However, for about 40 years, $D$. dianthicola has been associated with wilt and necrosis in potato plants in Europe, and thus, the European isolates of $D$. dianthicola were considered to be adapted to a cooler climate.

During the last decade, Dickeya strains belonging to a new clade have been isolated from diseased potato plants in many countries. In a 2004-2005 survey, atypical Dickeya strains were identified in Finland from potato plants showing symptoms of stem rot and wilting and tubers showing rotting [5]. Similar Dickeya strains were identified after 2005 from symptomatic plants in several European countries [6], in Israel [7] and in Georgia [8]. These strains have been described as a novel species named Dickeya solani [9]. Similar strains have been identified from ornamental plants in the Netherlands, leading to the hypothesis that $D$. solani has moved from ornamental plants to potato in the Netherlands, and it is now spreading with potato seed tubers [10].

D. solani causes rotting of potato stems in the field and soft rot of tubers during storage [10]. Although these symptoms can be caused also by other soft rot Enterobacteria, mainly different Pectobacterium species and $D$. dianthicola, the disease caused by $D$. solani appears to be more aggressive and leads to damage under a wider range of conditions and at lower bacterial loadings. Furthermore, the bacterium is able to spread from soil into plant roots and vascular tissue very efficiently, which causes a high level of spreading during growing season [11]. In some countries, the new pathogen has replaced other Dickeya strains and Pectobacterium species previously dominant in infected plants, and it appears to be highly aggressive, especially in hot climate conditions, raising implications for the increased importance of this pathogen in response to global warming [12]. Furthermore, $D$. solani has been proposed to be less susceptible for antagonism by saprophytic bacteria in the potato ecosystem [12]. In addition to D. solani, also a new Pectobacterium species, P. wasabiae, has been recently identified in Europe [13].

In order to understand what gives $D$. solani its biological properties, we sequenced the genome of a Finnish D. solani strain isolated from diseased potato stem. The analysis of the D. solani genome content highlighted the mosaic structure of this pathogen and revealed open reading frames (ORFs) possibly involved in virulence and the production of toxic compounds. 


\section{Experimental Section}

\section{Genome Sequencing and Analysis}

The genome sequence of the D. solani s0432-1 strain was obtained using Roche 454 GS Flx Titanium chemistry [14]. A total of 261,916 fragment library reads (an average length of $356 \mathrm{bp}$ ) containing 93,253,204 bps were assembled using Newbler (Roche 454 Life Science, software release 2.0.00.20), which generated 98 contigs ( $\geq 100 \mathrm{bp}$ ), including 4,873,690 bps at $\sim 19$-fold coverage. Gaps were filled by sequencing PCR or linker-PCR products using an ABI 3730 capillary sequencer (Applied Biosystems, Foster, CA, USA). Inferring the orientation and the order of the obtained contigs, gap closings were done using the Gap4 program (Staden package) [15]. This Whole Genome Shotgun project has been deposited at DNA Data Bank of Japan (DDBJ)/European Molecular Biology Laboratory (EMBL)/GenBank under the accession AMWE00000000. The version described in this paper is the first version, AMWE01000000. The proteins have locus tags that start with A544_, but they have been omitted in this text for simplicity.

Gene prediction from the Dickeya sp. D s0432-1 genome was done by using the Prodical gene prediction program [16]. Results from the gene prediction were validated with the GenePRIMP program [17] and by manual curation. Predicted gene models were then translated into peptides and functionally annotated with the Protein ANNotation with Z-scoRE (PANNZER) tool [18]. The PANNZER tool predicts human readable descriptions about the function, as well as Gene Ontology (GO) classes and Enzyme Commission (EC) numbers. Peptide sequences were assigned into Cluster of Orthologous Groups of proteins (COG) database clusters by using COGnitor tool [19]. Ribosomal RNA genes were computationally predicted using RNAmmer 1.2 [20]. The analysis revealed the presence of 5S, 16S and 23S rRNA genes organized in a co-transcribed operon. There are 7 copies of these transcription units in the genome, and an additional copy of 5S rRNA is present as a duplicate in one of the rRNA operons. The Average Nucleotide Identity (ANI) was calculated with JSpecies [21] using Basic Local Alignment Search Tool (BLAST) nucleotides alignments, MUMmer, designed to compare large DNA stretches and avoid previous manipulation of the sequences, and Tetra, an alignment-free analysis based on oligonucleotide signature frequencies. Multiple genome alignments with contigs of Dickeya sp. D s0432-1 were done with Mauve v.2.3.1 [22] against the genome of Dickeya dadantii 3937.

D. solani-specific ORFs were identified by aligning protein sequences from D s0432-1 with those in protein databases. Contigs of $D$. solani D s0432-1 were reordered to match the genome of D. dadantii 3937. The genome of D. solani D s0432-1 was also aligned against genomes of Dickeya isolates, Ech586, Ech703 and Ech1591, and several Pectobacterium genomes. Strains Ech586, Ech703 and Ech1591 (formerly D. dadantii, D. dadantii and D. zeae, respectively) have been re-classified, and the new names are D. zeae, D. paradisiaca and D. chrysanthemi, respectively [23]. BlastN alignment of $D$. solani genome to the National Center for Biotechnology Information (NCBI) nucleotide database was utilized to identify nucleotide sequences that were specific to D. solani D s0432-1. The obtained sequences were aligned to a protein database (BlastX, expected threshold of 10). Most of the nucleotide sequences that were considered to be specific at the nucleotide level showed similarity with known and predicted proteins of Dickeya or Pectobacterium isolates when aligned to the protein 
database. Only the genes that had the highest similarity to genes present in other bacteria (not Dickeya or Pectobacterium) were characterized further.

The improved annotation of the PKS/FAS/NRPS clusters was based on the Position-Specific Iterated BLAST (PSI-BLAST) protein search (including conserved domain search) [24] and on the InterProScan Sequence Search [25]. The substrate specificity conferring amino acids of the adenylation domains as defined by Stachelhaus [26] were found using a prediction program for PKS/NRPS [27,28] and by aligning with the GrsA adenylation domain [26] Gene islands were predicted combining SIGIHMM, PAI-IDA and IslandViewer tools, and their borders were manually adjusted considering genes in operons and accessory element often associated with mobile genetic elements, such as bacteriophages, insertion sequence elements, transposase, integrase, tRNA genes and repeat sequences [29]. The presence of $D$. solani genomic islands in other bacteria was also investigated by comparing the nucleotide sequences of the islands against the BlastN database.

\section{Results and Discussion}

\subsection{Sequence Comparisons of D. solani to Other Dickeya Strains}

A genomic sequence of the D. solani strain D s0432-1 was obtained with 454 sequencing. The length of the sequence was in total $4.9 \mathrm{Mb}$. Three gaps remained in the genome, and in spite of numerous attempts, the gaps could not be closed with PCR. BLAST analysis with the terminal sequences of the gaps revealed that the missing sequence in the first gap was located within a gene coding for large multi-repeat adhesin [30] that was annotated thus as two open reading frames (ORFs 875 and 876), and the second and third gaps were within genes having homology to hemolysins and cdiA genes, linked to contact-dependent growth inhibition in other bacteria [31]. The second genomic area of missing sequence resulted in two ORFs (2196 and 2197) and the third was within one gene (2274). Presumably, the repetitive nature of these genes makes them difficult to assemble.

Average Nucleotide Identity (ANI) was calculated with JSpecies, MUMmer and Tetra to identify the most similar Dickeya species among the strains for which the genome sequence was available. All algorithms rendered similar results, suggesting D. dadantii 3937 to have the greatest sequence similarity to D. solani s0432-1 based on nucleotide identity (Table 1). Even the analysis with the pairwise alignment tool Mauve, showed close synteny between the genomes of D s0432-1 and D. dadantii 3937 (Figure 1). This is in agreement with previous results with DNA-DNA hybridization showing that D. solani strains are most similar to D. dadantii [9].

\subsection{Manual Identification of Known Virulence Determinants}

The annotation of the D s0432-1 genome identified 4173 protein coding genes. There were 10 genes coding for pectate lyases. Six were annotated as pectin-modifying enzymes, and one gene was annotated as exopolygalacturonate lyase and one as galacturan 1,4-alpha-galacturonidase (Supplementary Table S1). Furthermore, the strain has the genes needed for the uptake and catabolism of pectic substrates [32]. Two endo-1,4-beta-glucanase genes coding for cellulases, celY and celZ, were also evident [33]. Among the annotated genes, numerous ORFs were annotated as proteases that are either secreted, membrane bound or periplasmic or function in the proteasome, including the ClpXP 
regulating Type III secretion [34]. Four metalloproteases annotated as serralysins were located adjacent to protease secretion genes and the protease inhibitor preventing the activity of the proteases before secretion [35]. The strain also has the necrosis-inducing protein toxin [36,37] and two copies of a putative avirulence protein, named $a v r L$ and $a v r M$, present also in other Dickeya species [38].

Table 1. Average Nucleotide Identity (ANI) calculated based on comparisons between D. solani D s0432-1 and other published Dickeya genomes. Pectobacterium atrosepticum SCRI1043 is included as a non-Dickeya pathogen for comparison.

\begin{tabular}{ccccccc}
\hline & $\begin{array}{c}\text { D. solani D } \\
\text { s0432-1 }\end{array}$ & $\begin{array}{c}\text { D. dadantii } \\
\mathbf{3 9 3 7}\end{array}$ & $\begin{array}{c}\text { D. zeae } \\
\text { Ech586 }\end{array}$ & $\begin{array}{c}\text { D. paradisiaca } \\
\text { Ech703 }\end{array}$ & $\begin{array}{c}\text { D. chrysanthemi } \\
\text { Ech1591 }\end{array}$ & $\begin{array}{c}\text { P. atrosepticum } \\
\text { SCRI1043 }\end{array}$ \\
\hline $\begin{array}{c}\text { D. solani } \\
\text { D s0432-1 }\end{array}$ & --- & 94 & 85 & 79 & 86 & 75 \\
$\begin{array}{c}\text { D. dadantii } \\
\quad 3937\end{array}$ & 94 & --- & 85 & 79 & 87 & 75 \\
$\quad \begin{array}{l}\text { D. zeae } \\
\text { Ech586 }\end{array}$ & 85 & 85 & --- & 78 & 86 & 74 \\
$\begin{array}{c}\text { D. paradisiaca } \\
\text { Ech703 }\end{array}$ & 79 & 79 & 78 & -- & 79 & 74 \\
$\begin{array}{c}\text { D. chrysanthemi } \\
\text { Ech1591 }\end{array}$ & 86 & 87 & 86 & 79 & -- & 75 \\
$\begin{array}{c}\text { P. atrosepticum } \\
\text { SCRI1043 }\end{array}$ & 75 & 75 & 74 & 74 & 75 & -- \\
\hline
\end{tabular}

Figure 1. Synteny of the D. solani D s0432-1 genome and genomes of other Dickeya strains. Pairwise alignments of genomes were generated using Mauve. Colored outlined blocks surround the regions of the genomic sequence that aligned to another genome. The colored bars inside the blocks are related to the level of sequence similarities. The analysis showed that the lowest number of rearrangements was evident between D. solani D s0432-1 and Dickeya dadantii 3937.

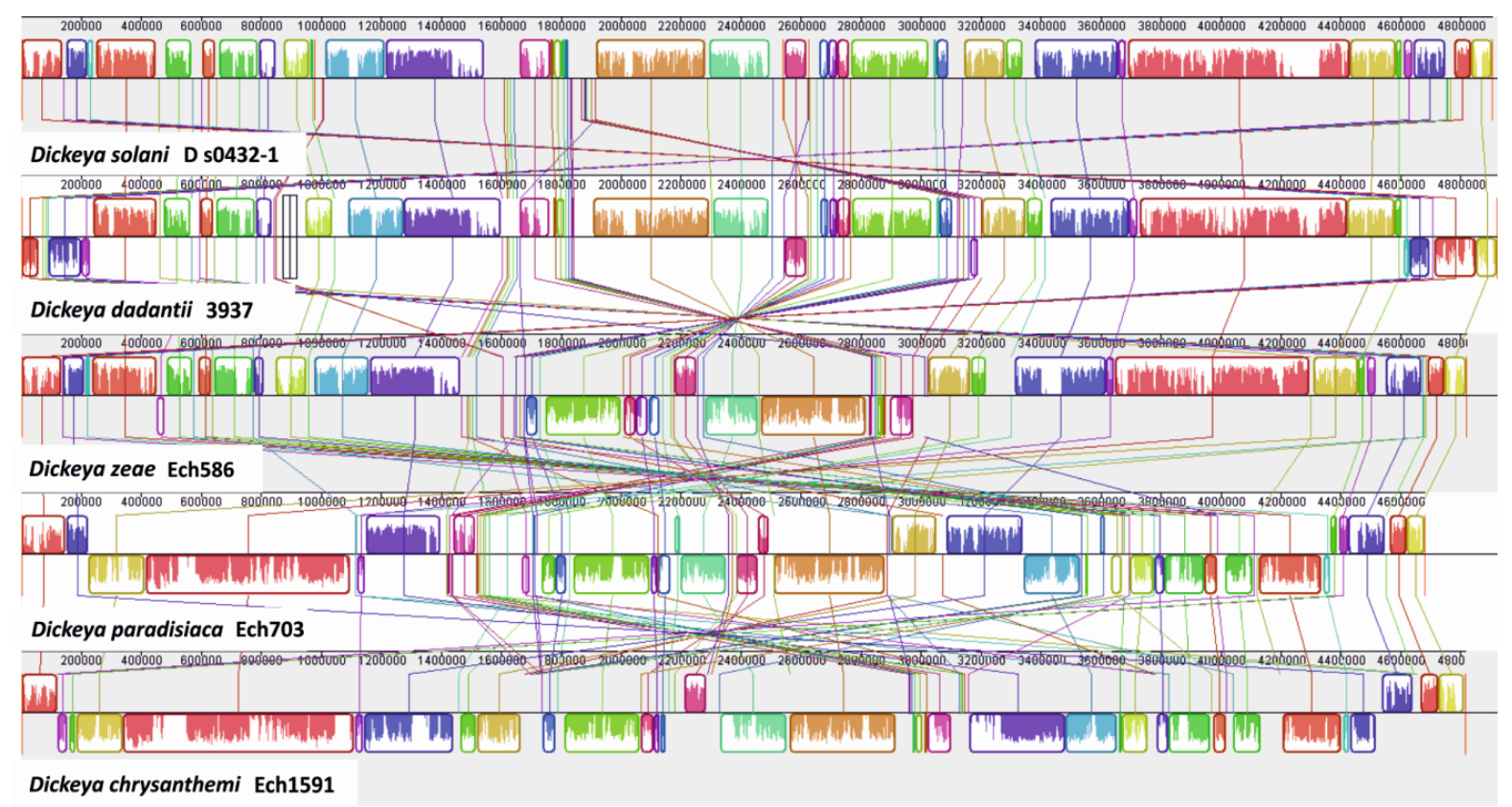


All the sequenced Dickeya strains have many genes that are related to sequestration or storage of iron (Table S1) [39]. D. solani D s0432-1 has genes for the production of two known siderophores, chrysobactin and achromobactin, which are needed for the virulence in $D$. dadantii. Furthermore, even the other iron-related genes, ftnA and $b f r$, coding for ferritin and bacterioferritin, respectively, and two genes coding for ferritin-like Dps proteins, are similar as in the characterized Dickeya strains [40,41]. Furthermore, D. solani D s0432-1 has the opgG and opgH genes for the production of periplasmic glucans [42] and sapA-sapC genes conferring resistance to antimicrobial peptides [43]. Similar to D. dadantii, the D s0432-1 strain has also the four genes coding for insect-specific Cyt-like toxin causing septicemia and death in pea aphids [44].

Genes coding for the components of all the six secretion systems identified in soft rot bacteria [45] were present also in the genome of $D$. solani D s0432-1 (Table S1). The effectors of Type VI secretion (T6SS), hemolysin-coregulated proteins (Hcp), were encoded by seven genes some adjacent to genes with similarity to genes coding for VgrG and Rhs. As in other soft rot bacteria, one copy of Hcp and $\operatorname{VgrG}$ was present in the T6SS cluster [46]. D. solani D s0432-1 also has the genes for the production of indigoidine, a blue pigment produced by all Dickeya strains [45]. It also contains the $v \mathrm{fm}$ gene cluster coding for a new quorum sensing system identified in D. dadantii [47].

Comparison of virulence regulators between $D$. solani D s0432-1 and $D$. dadantii revealed no differences. The D s0432-1 strain harbored the known virulence genes regulating the enzyme production, T3SS and production of extracellular polysaccharides and indigoidine [45]. Furthermore, the regulators, pir [48], rsmA and $r s m C$ genes, and the regulatory RNA $r s m B$ (genomic coordinates $1,057,799-1,058,066)$ could be found in the genome. The T6SS cluster contained a vasH homologue, suggesting that also T6 secretion is regulated by a similar mechanism as in Pectobacterium species. In conclusion, D. solani strain D s0432-1 seems to harbor most, if not all, the virulence determinants characterized previously in Dickeya isolates.

\subsection{Identification of Large Genomic Regions in the D. solani Genome, Likely Acquired by} Lateral Transmission

D. solani D s0432-1 nucleotide sequence were compared, using BLAST, to nine Dickeya and Pectobacterium genomes to identify similarities and differences between D. solani and other sequenced Dickeya and Pectobacterium isolates. It was evident that D. solani strain D s0432-1 shares large parts of its genome with D. dadantii 3937 and other Dickeya strains (Figure 2). However, some parts of the D s0432-1 genome have a similar sequence only in one or a few Dickeya strains. 
Figure 2. Comparative genomics between Dickeya strains. Comparison of gene coding sequences between D. solani D s0432-1 and other sequenced Dickeya strains reveals three large genomic regions (1, 2 and 3) coding for non-ribosomal peptide synthetases and polyketide synthetases (numbered according to the order of description in the results). The tentative $D$. solani-specific open reading frames are shown as the outermost ring.

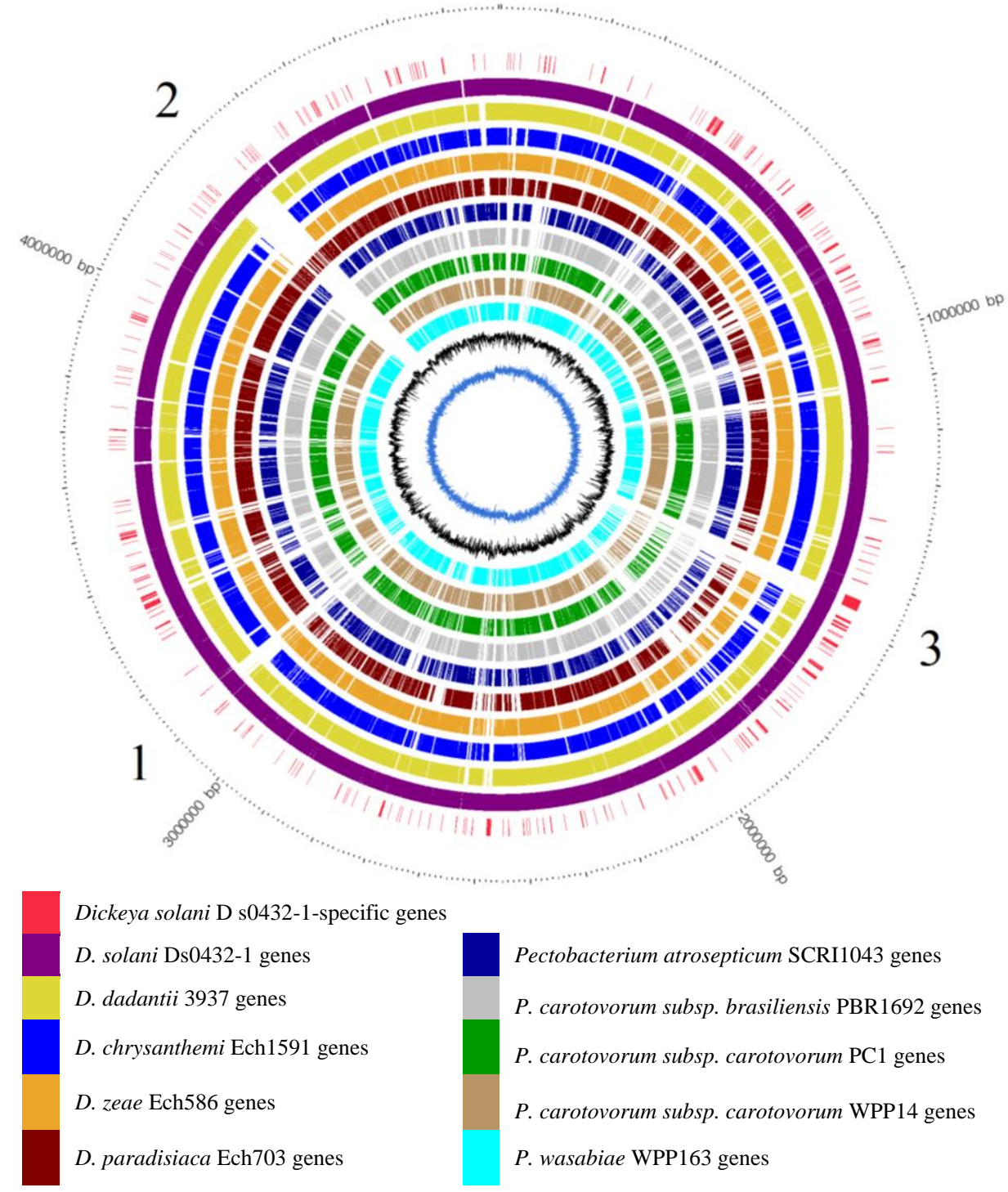

Genomic sequence of 41,546 nucleotides (genomic coordinates 3,092,397-3,133,943, region 1 in Figure 2), annotated to code for 12 proteins (ORFs 2680-2691), showed significant similarity to the sequence in Dickeya strain Ech586, but not to the other Dickeya strains or other soft rot bacteria. The organization of the genes in the clusters of Ech586 and D. solani were similar (Figure 3), the protein sequences ranging from $76 \%$ to $97 \%$ identity. Similarity with this gene cluster was also found in the genome of Teredinibacter turnerae T7901, an intracellular endosymbiont of marine wood-boring shipworms [49] (Figure 3), but the amino acid sequence similarities between D. solani and T. turnerae were lower ranging, from $39 \%$ to $72 \%$ identity, and only part of the D. solani cluster (ORFs 2691-2687) coding for PKS-related genes showed similarity to the $T$. turnerae sequence. Some of the ORFs were annotated as polyketide synthases (PKS), non-ribosomal peptide synthetases (NRPS) and amino acid adenylation domain proteins, suggesting that they code for proteins that may 
be involved in the production of antibiotics, toxins, siderophores or other secondary metabolites. PKSs and NRPSs produce molecules by sequential condensation of short carboxylic acids and amino acids, respectively. PKSs and NRPSs are thiotemplate modular systems (TMS): both direct product formation on a protein template by maintaining reaction intermediates covalently bound as thioesters on the same phosphopantetheine prosthetic group. The synthesized molecules can have antibiotic and phytotoxic effects and are found to increase the virulence in bacterial plant pathogens [50]. In this operon, PKS and NRPS modules are combined together to form a PKS/NRPS hybrid system that might be able to produce compounds of even greater chemical structural diversity compared to the ones produced by the single PKS and NRPS [51]. The D. solani ORF 2691 codes for a large multidomain protein, a type I PKS, where no acyltransferase (AT) domain could be identified; instead, an ORF 2687 may code for a free-standing acyl transferase protein, suggesting that ORF 2691 codes for a trans-AT PKS [52]. The polyketide may start with a fatty acid or a polyketide part of an unknown length, because the first domain of the PKS is keto-synthase (KS), which is usually a condensation domain, indicating that there is an undetected loading protein somewhere providing an unknown acyl precursor to the biosynthesis start. Furthermore, the cluster contains a separate dehydratase, acyl carrier protein and acyl transferase-acyl carrier protein and three NRPSs. The last NRPS carries the thioesterase domain at the end, which hydrolyzes the completed polypeptide chain from the previous module, ensuring termination. The sequences of the three NRPSs suggest that the synthetized molecule may contain glycine, serine and valine or isoleucine. The function and exact structure of this metabolite produced by $D$. solani and Ech586 is unknown.

A second, very large genomic sequence of 81,060 nucleotides (genomic coordinates 4,246,1944,327,254, region 2 in Figure 2) annotated to code for 25 genes (ORFs 3637-3661) showed similarity to the genome of D. paradisiaca Ech703, but not to the other Dickeya strains or other soft rot bacteria. This area of the D s0432-1 chromosome contained 13 ORFs annotated as polyketide synthase modules and acyl carrier proteins and other genes related to the production of secondary metabolites (Figure 4). Some of the ORFs are very large, the largest being ORF 3649, encoding a protein with a size of 6078 amino acids. In addition to $D$. paradisiaca Ech 703, high similarity throughout the whole gene cluster was observed also in Serratia odorifera 4Rx13, an antagonist of fungal pathogen Rhizoctonia solani [53] isolated from the rhizosphere of potato, which may suggest that the Dickeya species have obtained this cluster from soil bacteria. The similarity between the D. solani cluster and a similar cluster in Ech703 varies from $79 \%$ to $100 \%$ identity, and the organization of the cluster was very similar in Dickeya strains and in Serratia odorifera (Figure 4). Recently, this genomic area of Dickeya Ech703 and similar gene clusters in S. marcescens and S. plymuthica have been shown to code for trans-AT PKSs involved in the synthesis of oocydin A, a halogenated macrolide with broad-spectrum antifungal and anti-oomycete activity [54]. The homology of the protein sequences and the similar organization of the ORFs suggest that even $D$. solani D s0432-1 may produce oocydin A-like molecules. 
Figure 3. Alignment of the PKS/NRPS cluster at genomic coordinates of 3092397-3133943 in D. solani D s0432-1 with the two closest homologs in D. zeae Ech586 and T. turnerae T7901. The improved annotations of the genes, domains, substrate specificity conferring amino acids of the adenylation domains of NRPSs and predicted amino acids in the synthetized molecules are mentioned in the table within the figure. The colors of open reading frames (ORFs) indicate their functions: red for NRPS, green for PKS, grey for transport and yellow for tailoring. For PKS, the following domain abbreviations are used: acyl carrier protein (ACP), ketoacyl synthase (KS), ketoreductase $(\mathrm{KR})$ and dehydratase (DH). For NRPS, the domains are: adenylation (A), condensation (C), oxidation (Ox), thioesterase (Te) and 4-phosphopantetheine attachment site (PP).

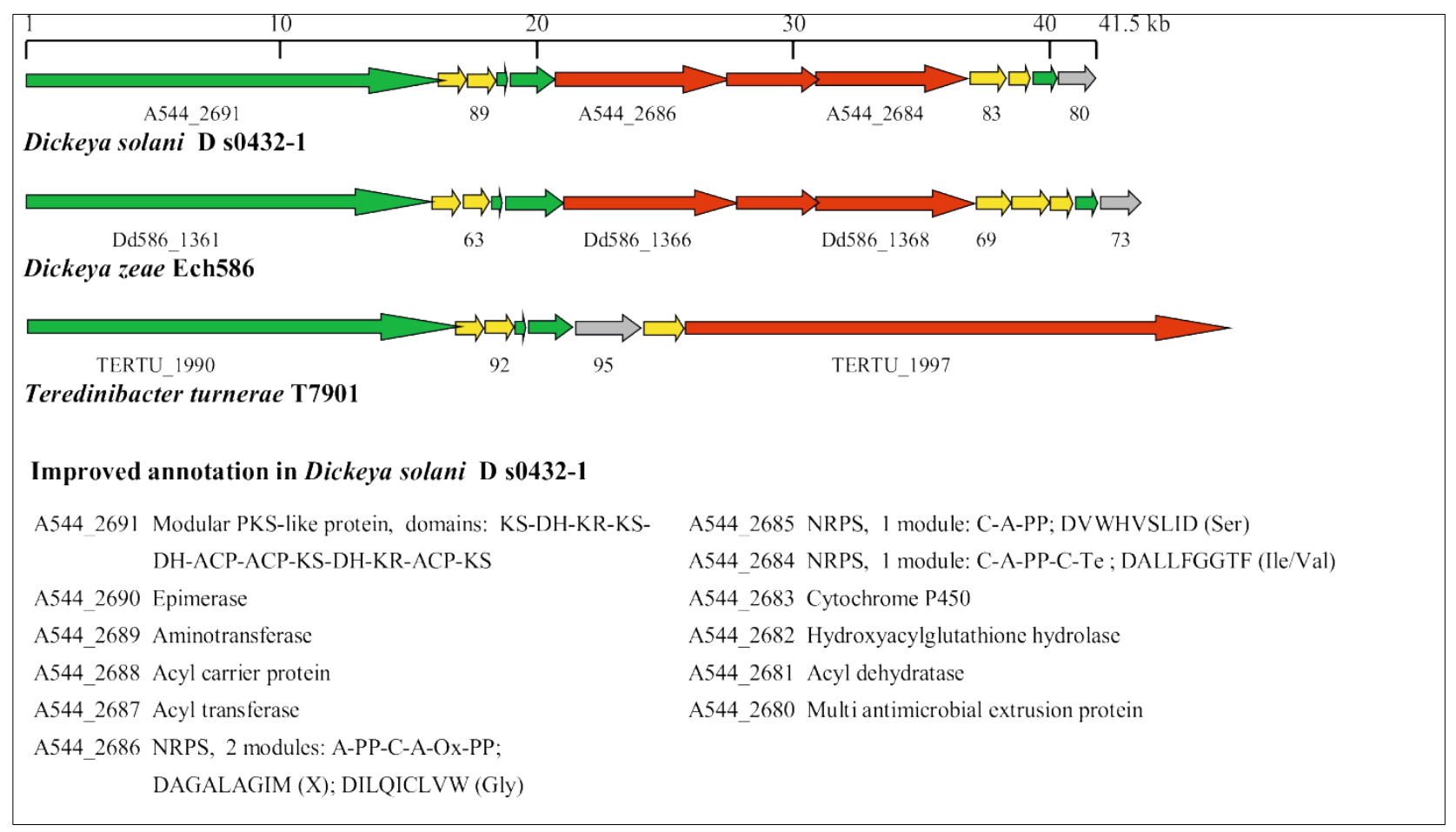

\subsection{Identification of D. solani s0432-1-Specific ORFs}

BlastN alignment of $D$. solani genome to the NCBI nucleotide database was utilized to identify nucleotide sequences that were present in D. solani D s0432-1, but not present in the other nine analyzed soft rot bacteria (Supplementary table S2). Many unique ORFs without significant similarity within Dickeya and Pectobacterium species were identified (Figure 2). Most of the identified sequences were small, single ORFs scattered throughout the genome or ORFs starting or ending at different nucleotides when compared to the other Dickeya genomes. These differences were interpreted as differences in the annotation process rather than real differences in the sequence. However, 57 genes were interpreted to be clearly not present in the other Dickeya and Pectobacterium species used in the comparison (Supplementary table S2). Many of the ORFs were hypothetical or poorly characterized in other organisms, and even in D. solani, their function was impossible to identify. These genes are not described further. 
Figure 4. Alignment of the PKS cluster (genomic coordinates 4,246,194-4,327,254) of D. solani D s0432-1, with the two closest homologs in D. paradisiaca Ech703 and S. odorifera 4Rx13 SODb. The improved annotations of the genes, domains, substrate specificity conferring amino acids of the adenylation domains of NRPSs and predicted amino acids in the synthetized molecules are mentioned in the table within the figure. The colors of ORFs indicate their functions: green for PKS, blue for FAS-like, grey for transport, yellow for tailoring and white for hypothetical. For PKS, the following domain abbreviations are used: acyl carrier protein (ACP), ketoacyl synthase (KS), ketoreductase (KR), dehydratase (DH), haloacid dehalogenase (HAD), methyltransferase (MT) and unknown $(\mathrm{X})$.

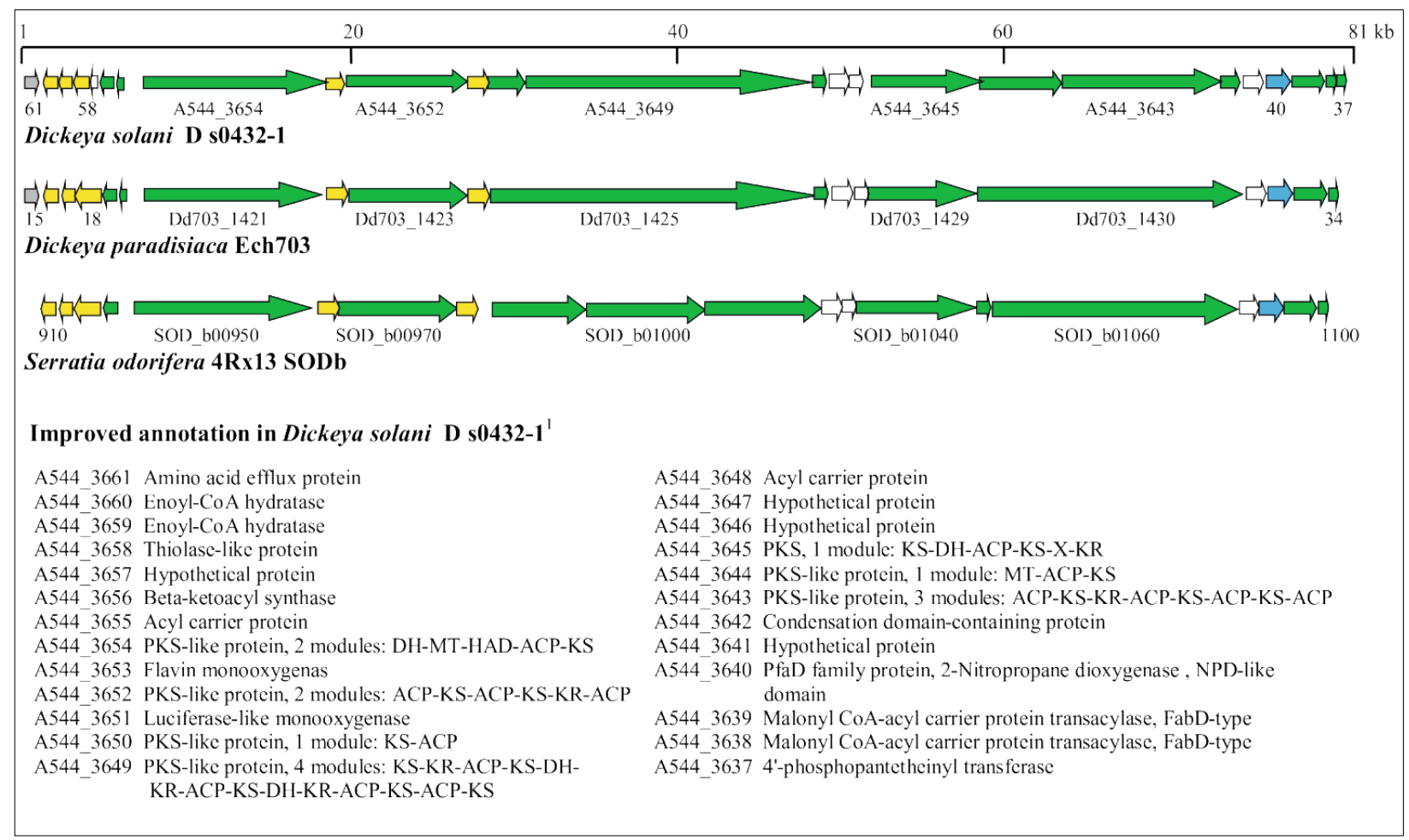

Among the identified D. solani-specific sequences, ORFs 1369-1388, corresponding to nucleotides 1,546,840-1,602,683 (region 3 in Figure 2), contained several large genes that were annotated as PKS, fatty acid synthases (FAS) and NRPS (Figure 5). The closest similarity of ORFs 1369-1386 was to Serratia plymuthica strains AS12 and A30, which are antagonists and growth-promoting soil bacteria [12,55]. Within this D. solani genomic region, the ORF 1374 showed similarity to the published $5^{\prime}$ end of $z m s A$, coding for a novel multidomain polyketide synthase that is essential for the production of zeamine, a novel polyamino-amide antibiotic of Dickeya zeae DZ1 [56,57]. Furthermore, a similar genomic cluster has been characterized from $S$. plymuthica RVH1 and was shown to lead to the biosynthesis of zeamine [58]. Unfortunately, the sequences of these clusters were not found in the databases, preventing a direct homology comparison. However, the organization of the ORFs, the domains within the ORFs and the predicted amino acids in the predicted product of the $D$. solani FAS/PKS/NRPS cluster are very similar to the $S$. plymuthica RVH1 zeamine cluster and zeamine, suggesting that $D$. solani $\mathrm{D}$ s0432-1 may synthesize a molecule with similarity to zeamine. The combination of ORFs suggests that this is a second PKS/NRPS hybrid system present in D. solani 
and lacking from $D$. dadantii [51]. Although NRPS modules are usually conserved, the KS domain of the PKS module in ORF 1374 seems to be unique in this hybrid, and it was not found in any other Pectobacterium or Dickeya spp. Furthermore, the other specific genes with features similar to PKS modules might be modules with novel functions and/or inter-modular linkers facilitating the transfer of the growing polyketide intermediate between PKS and NRPS modules in the hybrid system [59]. ATP-binding cassette $(\mathrm{ABC})$ transporter genes were found adjacent to the PKS/NRPS modules within the cluster. In Gram-negative bacteria, $A B C$ transporters can function as importers or exporters of toxins, bacteriocins, proteases and lipases. This ABC-type multidrug transport system consists of two inner membrane proteins, an ATPase, a membrane fusion protein and an outer membrane polypeptide. Furthermore, two ORFs (1387-1388) residing outside the conserved region at the end of the cluster were annotated as multidrug efflux proteins and may be part of the FAS/PKS/NRPS cluster in D. solani, even if they are lacking from the Serratia genomes. The presence of ORFs annotated as transporters next to the PKS/NRPS modules may suggest that the synthesized product is secreted [60].

Figure 5. Alignment of the third FAS/PKS/NRPS cluster (genomic coordinates 1,546,840-1,602,683) of D. solani D s0432-1 with the two closest homologs in Serratia sp. AS12 and S. plymuthica A30. The improved annotations of the genes, domains, substrate specificity conferring amino acids of the adenylation domains of NRPSs and predicted amino acids in the synthetized molecules are mentioned in the table within the figure. The colors of ORFs indicate their functions: red for NRPS, green for PKS, blue for FAS-like, grey for transport, yellow for tailoring and white for hypothetical. For PKS, the following domain abbreviations were used: acyl carrier protein (ACP), acyltransferase (AT), ketoacyl synthase (KS) and ketoreductase (KR). For NRPS, the domains were: adenylation (A), condensation (C), epimerization (E) and 4-phosphopantetheine attachment site (PP).

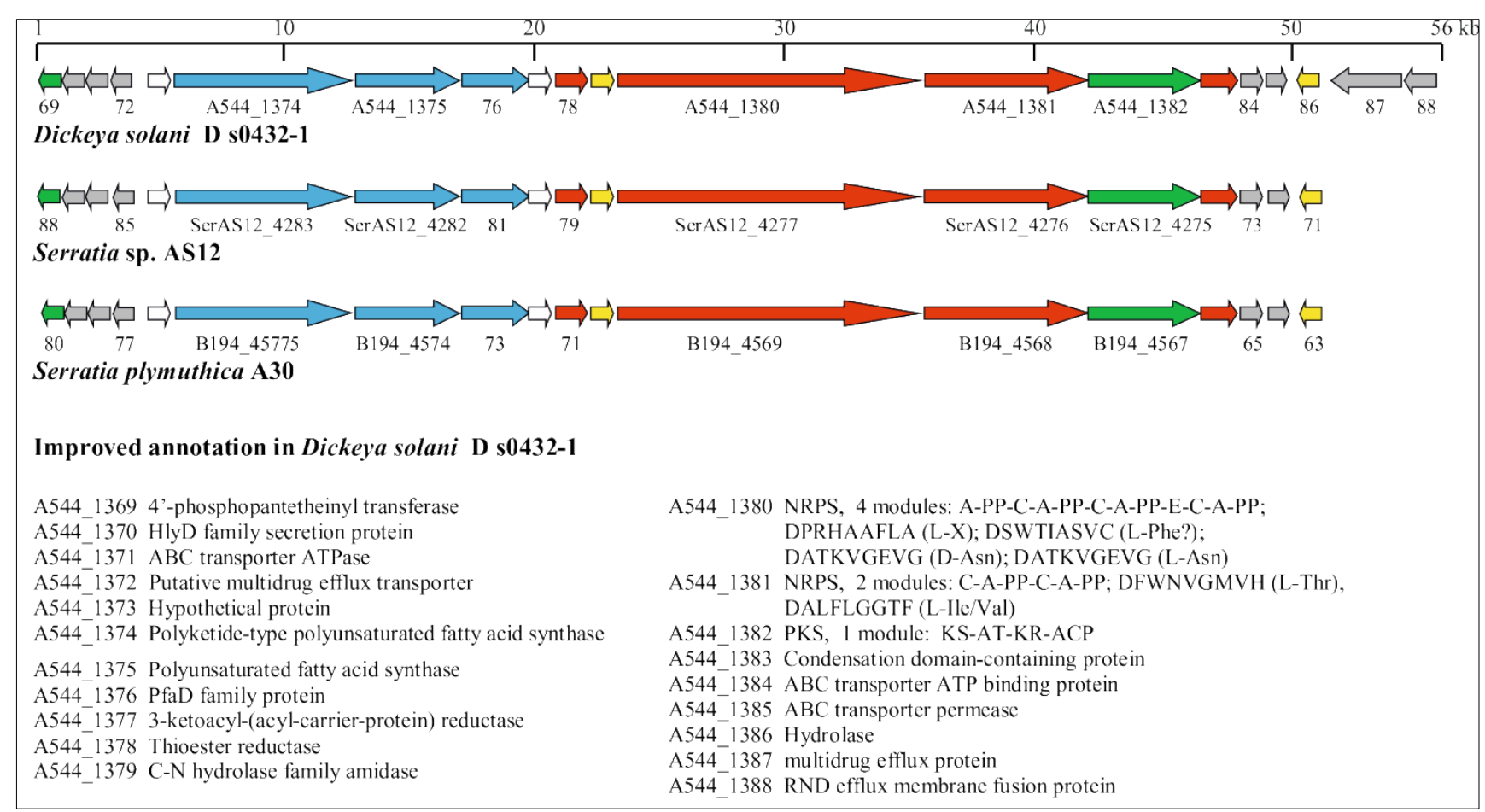


Among the remaining $D$. solani-specific ORFs, also a tripartite multidrug efflux system composed of the AcrB/AcrD/AcrF family cation/multidrug efflux pump of the Resistance-Nodulation-Cell Division (RND type (ORF 978) and two efflux transporters (ORFs 979 and 980) were identified. RND transporters have broad substrate specificity and, unlike the other multidrug efflux pumps, they pump out the drug molecules directly into the external medium rather than into the periplasm. RND-type pumps have been shown to confer antibiotic resistance in Gram-negative bacteria. In plant pathogenic bacteria, AcrAB-type pumps play a defensive role against new inhibitors, allowing the bacteria to survive and enabling them to evolve specific defense mechanisms for the new environment [61]. Multidrug efflux systems have been shown to protect Agrobacterium tumefaciens, Erwinia amylovora and Pseudomonas syringae against antibiotics and detergents [62,63], while in D. dadantii 3937, similar efflux genes have been shown to be involved in resistance against plant antibacterial peptides [64]. At the DNA level, the D. solani-specific efflux system genes showed the highest similarity to genes in Sinorhizobium fredii (pump) or A. vitis (transporters), but less similar proteins were present in many bacteria, including other Dickeya strains.

Among the ORFs identified as specific to D. solani D s0432-1, several were annotated as coding for proteins that interact with DNA. Two groups of genes, each containing three genes (ORFs 412-414 and 639-641), code for components of type I restriction-modification (R-M) systems that protect bacteria from invading foreign DNA, such as bacteriophages. One of the R-M systems is a mosaic containing two ORFs (412 and 414) similar to the R-M system genes of Pectobacterium wasabiae SCC3193 and one gene (413) similar to a gene in Shewanella frigidimarina NCIMB 400, a marine bacterium. The second cluster was similar to R-M systems in E. coli and many other Enterobacteria. The R-M systems represent an advantage for a bacterium that is trying to colonize new ecological niches: they confer protection from potentially dangerous DNA acquisition, such as bacteriophages [65]. The presence of many R-M systems may explain why this bacterium is recalcitrant to genetic modifications, such as knock-out methods and transformation [66]. The remaining genes in this group were annotated or had COG descriptions as helicases (2161 and 2853) or as cytosine-C5-spesific DNA methylase (1622), possibly involved in reparation or modification of chromosomal DNA.

There were many enzyme encoding ORFs among the D. solani-specific sequences. Five D. solani-specific ORFs contained COG description or were annotated as demethylmenaquinone methyltransferases having a MenG domain (1392, 2872, 2974, 2975 and 3985). Demethylmenaquinone methyltransferase converts demethylmenaquinone to menaquinone as the terminal step in its biosynthesis. Demethylmenaquinone, menaquinone and quinone are electron carriers that participate in many bioenergetics chains. Most Gram-positive bacteria and anaerobic Gram-negative bacteria contain only menaquinone, whereas the majority of strictly aerobic Gram-negative bacteria contain exclusively ubiquinone [67]. Both types of isoprenoid quinones are found in facultative anaerobic Gram-negative bacteria, such as E. coli, where ubiquinone is used as the main quinone in aerobic respiration, whereas in anaerobic conditions, menaquinone and demethylmenaquinone are used as electron carriers [68]. In addition to the five MenG domain containing proteins, a sixth demethylmenaquinone methyltransferase is present in D. solani and all the other Dickeya genomes. The D. solani-specific demethylmenaquinone methyltransferases show similarity to proteins present in many bacteria and fungi, among them many environmental isolates and isolates having interactions with plants. In Mesorhizobium huakuii, a demethylmenaquinone methyltransferase has been shown to accumulate 
under microaerobic conditions and to be necessary for the bacteroid development [69]. The presence of many $D$. solani-specific OFRs possibly involved in the synthesis of menaquinone may suggest that it differs from the other Dickeya species in its respiration under anaerobic conditions. Some D. solani-specific genes encode proteins that may be involved in the degradation of peptides, amino acids or organic molecules. A subtilisin-like peptidase (1265) with the highest similarity in many Xanthomonas species was identified as a D. solani-specific gene. D-serine deaminase (722), aspartate/tyrosine/aromatic aminotransferase (1393) and aspartate aminotransferase A (2871) may affect the ability of $D$. solani to utilize amino acids.

Several of the D. solani-specific ORFs were annotated as regulators, particularly repressors. New transcriptional regulators may represent a short-term adaptive response affecting virulence gene expression in a new environment or host. Among the identified regulators, three genes (2972, 3986 and 1473) were annotated as lysR-like regulators, two of them adjacent to demethylmenaquinone methyltransferase genes and the third one (1473) next to a phospholipase that it may regulate. One of the repressors (1432) most likely codes for a regulator of adjacent metal ion uptake genes (1430 and 1431), and one of them (397) may originate from a phage. One of the D. solani-specific ORFs was annotated as an endoribonuclease-domain protein (720), showing similarity to putative translation initiation inhibitor $y j g F$. The $D$. solani-specific regulators showed the highest similarity to regulators in other bacteria, for example, in Burkholderia, Xylella or Vibrio, but less similar proteins were also present in other Dickeya strains, possibly due to the presence of other regulators within the same regulator gene families.

\subsection{Identification of Horizontally Acquired Genomic Islands in D. solani}

Horizontal gene transfer allows the acquisition of a number of accessory genes that might play an important role in promoting the adaptive evolution of bacteria [70]. These accessory genes cluster in genomic islands (GEI), which may influence antibiotic resistance or toxin production, symbiosis, fitness and other features that might be beneficial under certain environmental conditions. The identification of horizontally acquired islands revealed 20 GEIs that showed variable origins (Supplementary Table S3). Many of the D. solani islands contained common genes in bacteria, such as ribosomal genes, flagella and T2SS and showed the highest BlastN coverage in D. dadantii 3937, which suggests that these islands may be evolutionally old. Three of the GEIs were not present in $D$. dadantii 3937, suggesting that these islands may have been acquired recently by $D$. solani. One of them encoded a putative R-M system with homology to the D. chrysanthemi 1591 genome, while the other two GEIs (regions 2 and 3 in Figure 2) encoded reputed secondary metabolites. Furthermore, many $D$. solani GEIs present also in $D$. dadantii, and the other Dickeya genomes also harbored $D$. solani-specific ORFs not present in the other Dickeya isolates (Supplementary Table S2). These acquired genes in the genomic islands might function as hot spots for the insertion of new elements, highlighting the mosaic structure and fast evolution of genomic islands [71].

\section{Conclusions}

The genome of $D$. solani strain D s0432-1 suggests that this novel pathogen is most closely related to D. dadantii strain 3937 at the DNA level, as well as in the order of genes. Comparison of the ORFs 
present in $D$. solani and other Dickeya strains revealed that $D$. solani harbors a unique combination of large gene clusters possibly involved in the production and secretion of toxic secondary metabolites. It also contains ORFs coding for the efflux system and two restriction-modification systems possibly protecting the cells against toxins and bacteriophages (and genetic manipulations). Furthermore, the D. solani genome has several extra copies of genes that may be linked to the production of menaquinone, the major quinone used by facultative anaerobes in anaerobic conditions, possibly affecting its growth in anaerobic conditions. Many D. solani-specific ORFs were present in horizontally acquired gene islands that are also present in the other Dickeya species. The additional genes in these islands in D. solani suggest that the islands may be still evolving. It can be hypothesized that the novel genes and novel combination of gene clusters related to the production of secondary metabolites may be related to the high virulence, invasive character and replacement of common blackleg pathogens with D. solani in many European countries.

\section{Supplementary Materials}

Supplementary materials can be accessed at: http://www.mdpi.com/1424-2818/5/4/824/s1.

\section{Acknowledgments}

Comparative genomics and identification of $D$. solani-specific genes performed by Beijing Genome Institute are gratefully acknowledged. Johanna Nykyri is warmly thanked for help and for critically reading the manuscript. We gratefully acknowledge the financial support of the Academy of Finland (grant No. 128566), the University of Helsinki, Biocentrum Helsinki, Biocenter Finland, the Viikki Doctoral Programme in Molecular Biosciences, the Finnish Doctoral Programme in Computational Sciences and the Centre for International Mobility (CIMO), Finland.

\section{Conflicts of Interest}

The authors declare no conflict of interest.

\section{References and Notes}

1. Burkholder, W.; McFadden, L.; Dimock, A. A Bacterial Blight of Chrysanthemums. Phytopathology 1953, 43, 522-526.

2. Samson, R.; Legendre, J.; Christen, R.; Fischer-Le Saux, M.; Achouak, W.; Gardan, L. Transfer of Pectobacterium chrysanthemi (Burkholder et al. 1953) Brenner et al. 1973 and Brenneria paradisiaca to the Genus Dickeya Gen. Nov as Dickeya chrysanthemi Comb. Nov and Dickeya paradisiaca Comb. Nov and Delineation of Four Novel Species, Dickeya dadantii sp Nov., Dickeya dianthicola Sp Nov., Dickeya dieffenbachiae Sp Nov and Dickeya zeae Sp Nov. Int. J. Syst. Evol. Microbiol. 2005, 55, 1415-1427.

3. Ma, B.; Hibbing, M.E.; Kim, H.; Reedy, R.M.; Yedidia, I.; Breuer, J.; Breuer, J.; Glasner, J.D.; Perna, N.T.; Kelman, A.; et al. Host Range and Molecular Phylogenies of the Soft Rot Enterobacterial Genera Pectobacterium and Dickeya. Phytopathology 2007, 97, 1150-1163. 
4. Perombelon, M. Potato Diseases Caused by Soft Rot Erwinias: An Overview of Pathogenesis. Plant Pathol. 2002, 51, 1-12.

5. Laurila, J.; Ahola, V.; Lehtinen, A.; Joutsjoki, T.; Hannukkala, A.; Rahkonen, A.; Pirhonen, M. Characterization of Dickeya Strains Isolated from Potato and River Water Samples in Finland. Eur. J. Plant Pathol. 2008, 122, 213-225.

6. Slawiak, M.; van Beckhoven, J.R.C.M.; Speksnijder, A.G.C.L.; Czajkowski, R.; Grabe, G.; van der Wolf, J.M. Biochemical and Genetical Analysis Reveal a New Clade of Biovar 3 Dickeya Spp. Strains Isolated from Potato in Europe. Eur. J. Plant Pathol. 2009, 125, 245-261.

7. Tsror (Lahkim), L.; Erlich, O.; Lebiush, S.; Hazanovsky, M.; Zig, U.; Slawiak, M.; Grabe, G.; van der Wolf, J.M.; van de Haar, J.J. Assessment of Recent Outbreaks of Dickeya Sp (Syn. Erwinia chrysanthemi) Slow Wilt in Potato Crops in Israel. Eur. J. Plant Pathol. 2009, 123, 311-320.

8. Tsror (Lahkim), L.; Erlich, O.; Lebiush, S.; van der Wolf, J.; Czajkowski, R.; Mozes, G.; Sikharulidze, Z.; Ben Daniel, B. First Report of Potato Blackleg Caused by a Biovar 3 Dickeya Sp. in Georgia. New Disease Reports 2011, doi:10.5197/j.2044-0588.2011.023.001.

9. Van der Wolf, J.M.; Nijhuis, E.H.; Kowalewska, M.J.; Saddler, G.S.; Parkinson, N.; Elphinstone, J.G.; Pritchard, L.; Toth, I.K.; Lojkowska, E.; Potrykus, M.; et al. Dickeya solani Sp. Nov., a Pectinolytic Plant Pathogenic Bacterium Isolated from Potato (Solanum tuberosum). Int. J. Syst. Evol. Microbiol. 2013, doi: 10.1099/ijs.0.052944-0.

10. Toth, I.K.; van der Wolf, J.M.; Saddler, G.; Lojkowska, E.; Helias, V.; Pirhonen, M.; Tsror, L.; Elphinstone, J.G. Dickeya Species: An Emerging Problem for Potato Production in Europe. Plant Pathol. 2011, 60, 385-399.

11. Czajkowski, R.; de Boer, W.J.; Velvis, H.; van der Wolf, J.M. Systemic Colonization of Potato Plants by a Soilborne, Green Fluorescent Protein-Tagged Strain of Dickeya Sp Biovar 3. Phytopathology 2010, 100, 134-142.

12. Czajkowski, R.; de Boer, W.J.; van der Zouwen, P.S.; Kastelein, P.; Jafra, S.; de Haan, E.G.; van den Bovenkamp, G.W.; van der Wolf, J.M. Virulence of 'Dickeya solani' and Dickeya dianthicola Biovar-1 and -7 Strains on Potato (Solanum tuberosum). Plant Pathol. 2012, 62, 597-610.

13. Nykyri, J.; Niemi, O.; Koskinen, P.; Nokso-Koivisto, J.; Pasanen, M.; Broberg, M.; Plyusnin, I.; Törönen, P.; Holm, L.; Pirhonen, M.; et al. Revised Phylogeny and Novel Horizontally Acquired Virulence Determinants of the Model Soft Rot Phytopathogen Pectobacterium wasabiae SCC3193. PLoS Pathogens 2012, 8, e1003013.

14. Margulies, M.; Egholm, M.; Altman, W.; Attiya, S.; Bader, J.; Bemben, L.; Berka, J.; Braverman, M.; Chen, Y.; Chen, Z.; et al. Genome Sequencing in Microfabricated High-Density Picolitre Reactors. Nature 2005, 437, 376-380.

15. Staden, R.; Beal, K.F.; Bonfield, J.K. The Staden Package, 1998. Methods Mol. Bio. 2000, 132, 115.

16. Hyatt, D.; Chen, G.; LoCascio, P.F.; Land, M.L.; Larimer, F.W.; Hauser, L.J. Prodigal: Prokaryotic Gene Recognition and Translation Initiation Site Identification. BMC Bioinformatics 2010, 11, 119.

17. Pati, A.; Ivanova, N.N.; Mikhailova, N.; Ovchinnikova, G.; Hooper, S.D.; Lykidis, A.; Kyrpides, N.C. GenePRIMP: A Gene Prediction Improvement Pipeline for Prokaryotic Genomes. Nat. Methods 2010, 7, 455-462. 
18. Radivojac, P.; Clark, W.T.; Oron, T.R.; Schnoes, A.M.; Wittkop, T.; Sokolov, A.; Graim, K.; Funk, C.; Verspoor, K.; Ben-Hur, A.; et al. A Large-Scale Evaluation of Computational Protein Function Prediction. Nat. Methods 2013, 10 (suppl.), 221-227.

19. Tatusov, R.; Galperin, M.; Natale, D.; Koonin, E. The COG Database: A Tool for Genome-Scale Analysis of Protein Functions and Evolution. Nucleic Acids Res. 2000, 28, 33-36.

20. Lagesen, K.; Hallin, P.; Rodland, E.A.; Staerfeldt, H.; Rognes, T.; Ussery, D.W. RNAmmer: Consistent and Rapid Annotation of Ribosomal RNA Genes. Nucleic Acids Res. 2007, 35, 3100-3108.

21. Richter, M.; Rossello-Mora, R. Shifting the Genomic Gold Standard for the Prokaryotic Species Definition. Proc. Natl. Acad. Sci. U. S. A. 2009, 106, 19126-19131.

22. Darling, A.E.; Mau, B.; Perna, N.T. ProgressiveMauve: Multiple Genome Alignment with Gene Gain, Loss and Rearrangement. PLoS One 2010, 5, e11147.

23. Pritchard, L.; Humphris, S.; Saddler, G.S.; Parkinson, N.M.; Bertrand, V.; Elphinstone, J.G.; Toth, I.K. Detection of Phytopathogens of the Genus Dickeya using a PCR Primer Prediction Pipeline for Draft Bacterial Genome Sequences. Plant Pathol. 2013, 62, 587-596.

24. BLAST. Available online: http://blast.ncbi.nlm.nih.gov/blast.cgi (accessed on 8 March 2013).

25. InterProScan 4. Available online: http://www.ebi.ac.uk/tools/pfa/iprscan/ (accessed on 8 March 2013).

26. Stachelhaus, T.; Mootz, H.; Marahiel, M. The Specificity-Conferring Code of Adenylation Domains in Nonribosomal Peptide Synthetases. Chem. Biol. 1999, 6, 493-505.

27. PKS/NRPS Analysis Web-site. Available online: http://nrps.igs.umaryland.edu/nrps (accessed on 8 March 2013).

28. Bachmann, B.O.; Ravel, J. in Silico Prediction of Microbial Secondary Metabolic Pathways from DNA Sequence Data. Methods Enzymol. 2009, 458, 181-217.

29. Dobrindt, U.; Hochhut, B.; Hentschel, U.; Hacker, J. Genomic Islands in Pathogenic and Environmental Microorganisms. Nat. Rev. Microbiol. 2004, 2, 414-424.

30. Perez-Mendoza, D.; Coulthurst, S.J.; Humphris, S.; Campbell, E.; Welch, M.; Toth, I.K.; Salmond, G.P.C. A Multi-Repeat Adhesin of the Phytopathogen, Pectobacterium atrosepticum, is Secreted by a Type I Pathway and is Subject to Complex Regulation Involving a Non-Canonical Diguanylate Cyclase. Mol. Microbiol. 2011, 82, 719-733.

31. Aoki, S.K.; Diner, E.J.; de Roodenbeke, C.T.K.; Burgess, B.R.; Poole, S.J.; Braaten, B.A.; Jones, A.M.; Webb, J.S.; Hayes, C.S.; Cotter, P.A.; et al. A Widespread Family of Polymorphic Contact-Dependent Toxin Delivery Systems in Bacteria. Nature 2010, 468, 439-442.

32. Blot, N.; Berrier, C.; Hugouvieux-Cotte-Pattat, N.; Ghazi, A.; Condemine, G. The Oligogalacturonate-Specific Porin KdgM of Erwinia chrysanthemi belongs to a New Porin Family. J. Biol. Chem. 2002, 277, 7936-7944.

33. Aymeric, J.; Guiseppi, A.; Pascal, M.; Chippaux, M. Mapping and Regulation of the Cel Genes in Erwinia chrysanthemi. Mol. Gen. Genet. 1988, 211, 95-101.

34. Li, Y.; Yamazaki, A.; Zou, L.; Biddle, E.; Zeng, Q.; Wang, Y.; Lin, H.; Wang, Q.; Yang, C. ClpXP Protease Regulates the Type III Secretion System of Dickeya dadantii 3937 and is Essential for the Bacterial Virulence. Mol. Plant-Microbe Interact. 2010, 23, 871-878. 
35. Wandersman, C.; Delepelaire, P.; Letoffe, S. Secretion Processing and Activation of Erwinia chrysanthemi Proteases. Biochimie 1990, 72, 143-146.

36. Mattinen, L.; Tshuikina, M.; Mae, A.; Pirhonen, M. Identification and Characterization of Nip, Necrosis-Inducing Virulence Protein of Erwinia carotovora Subsp carotovora. Mol. PlantMicrobe Interact. 2004, 17, 1366-1375.

37. Ottmann, C.; Luberacki, B.; Kuefner, I.; Koch, W.; Brunner, F.; Weyand, M.; Mattinen, L.; Pirhonen, M.; Anderluh, G.; Seitz, H.U.; et al. A Common Toxin Fold Mediates Microbial Attack and Plant Defense. Proc. Natl. Acad. Sci. USA 2009, 106, 10359-10364.

38. Kazemi-Pour, N.; Condemine, G.; Hugouvieux-Cotte-Pattat, N. The Secretome of the Plant Pathogenic Bacterium Erwinia chrysanthemi. Proteomics 2004, 4, 3177-3186.

39. Expert, D. Withholding and Exchanging Iron: Interactions between Erwinia spp. and their Plant Hosts. Annu. Rev. Phytopathol. 1999, 37, 307-334.

40. Boughammoura, A.; Matzanke, B.F.; Boettger, L.; Reverchon, S.; Lesuisse, E.; Expert, D.; Franza, T. Differential Role of Ferritins in Iron Metabolism and Virulence of the PlantPathogenic Bacterium Erwinia chrysanthemi 3937. J. Bacteriol. 2008, 190, 1518-1530.

41. Boughammoura, A.; Expert, D.; Franza, T. Role of the Dickeya dadantii Dps Protein. Biometals 2012, 25, 423-433.

42. Bouchart, F.; Delangle, A.; Lemoine, J.; Bohin, J.; Lacroix, J. Proteomic Analysis of a NonVirulent Mutant of the Phytopathogenic Bacterium Erwinia chrysanthemi Deficient in Osmoregulated Periplasmic Glucans: Change in Protein Expression is Not Restricted to the Envelope, but Affects General Metabolism. Microbiology-(UK) 2007, 153, 760-767.

43. Lopez-Solanilla, E.; Garcia-Olmedo, F.; Rodriguez-Palenzuela, P. Inactivation of the sapA to sapF Locus of Erwinia chrysanthemi Reveals Common Features in Plant and Animal Bacterial Pathogenesis. Plant Cell 1998, 10, 917-924.

44. Costechareyre, D.; Balmand, S.; Condemine, G.; Rahbe, Y. Dickeya dadantii, a Plant Pathogenic Bacterium Producing Cyt-Like Entomotoxins, Causes Septicemia in the Pea Aphid Acyrthosiphon pisum. PLoS One 2012, 7, e30702.

45. Charkowski, A.; Blanco, C.; Condemine, G.; Expert, D.; Franza, T.; Hayes, C.; HugouvieuxCotte-Pattat, N.; Lopez Solanilla, E.; Low, D.; Moleleki, L.; et al. The Role of Secretion Systems and Small Molecules in Soft-Rot Enterobacteriaceae Pathogenicity. Annu. Rev. Phytopathol. 2012, 50, 425-449.

46. Mattinen, L.; Somervuo, P.; Nykyri, J.; Nissinen, R.; Kouvonen, P.; Corthals, G.; Auvinen, P.; Aittamaa, M.; Valkonen, J.P.T.; Pirhonen, M. Microarray Profiling of Host-Extract-Induced Genes and Characterization of the Type VI Secretion Cluster in the Potato Pathogen Pectobacterium atrosepticum. Microbiology-(UK) 2008, 154, 2387-2396.

47. Nasser, W.; Dorel, C.; Wawrzyniak, J.; Van Gijsegem, F.; Groleau, M.; Déziel, E.; Reverchon, S. Vfm a New Quorum Sensing System Controls the Virulence of Dickeya dadantii. Environ. Microbiol. 2013, 15, 865-880.

48. Nomura, K.; Nasser, W.; Tsuyumu, S. Self-Regulation of Pir, a Regulatory Protein Responsible for Hyperinduction of Pectate Lyase in Erwinia chrysanthemi EC16. Mol. Plant-Microbe Interact. 1999, 12, 385-390. 
49. Yang, J.C.; Madupu, R.; Durkin, A.S.; Ekborg, N.A.; Pedamallu, C.S.; Hostetler, J.B.; Radune, D.; Toms, B.S.; Henrissat, B.; Coutinho, P.M.; et al. The Complete Genome of Teredinibacter turnerae T7901: An Intracellular Endosymbiont of Marine Wood-Boring Bivalves (Shipworms). PLoS One 2009, 4, e6085.

50. Donadio, S.; Monciardini, P.; Sosio, M. Polyketide Synthases and Nonribosomal Peptide Synthetases: The Emerging View from Bacterial Genomics. Nat. Prod. Rep. 2007, 24, 1073-1109.

51. Shen, B.; Du, L.; Sanchez, C.; Edwards, D.J.; Chen, M.; Murrell, J.M. The Biosynthetic Gene Cluster for the Anticancer Drug Bleomycin from Streptomyces verticillus ATCC15003 as a Model for Hybrid Peptide-Polyketide Natural Product Biosynthesis. J. Ind. Microbiol. Biotechnol. 2001, 27, 378-385.

52. Piel, J. Biosynthesis of Polyketides by Trans-AT Polyketide Synthases. Nat. Prod. Rep. 2010, 27, 996-1047.

53. Kai, M.; Effmert, U.; Berg, G.; Piechulla, B. Volatiles of Bacterial Antagonists Inhibit Mycelial Growth of the Plant Pathogen Rhizoctonia solani. Arch. Microbiol. 2007, 187, 351-360.

54. Matilla, M.A.; Stoeckmann, H.; Leeper, F.J.; Salmond, G.P.C. Bacterial Biosynthetic Gene Clusters Encoding the Anti-Cancer Haterumalide Class of Molecules: Biogenesis of the Broad Spectrum Antifungal and Anti-Oomycete Compound, Oocydin A. J. Biol. Chem. 2012, 287, 39125-39138.

55. Neupane, S.; Finlay, R.D.; Alstrom, S.; Goodwin, L.; Kyrpides, N.C.; Lucas, S.; Lapidus, A.; Bruce, D.; Pitluck, S.; Peters, L.; et al. Complete Genome Sequence of Serratia plymuthica Strain AS12. Stand. Genomic Sci. 2012, 6, 165-173.

56. Wu, J.; Zhang, H.; Xu, J.; Cox, R.J.; Simpson, T.J.; Zhang, L. C-13 Labeling Reveals Multiple Amination Reactions in the Biosynthesis of a Novel Polyketide Polyamine Antibiotic Zeamine from Dickeya zeae. Chem. Commun. 2010, 46, 333-335.

57. Zhou, J.; Zhang, H.; Wu, J.; Liu, Q.; Xi, P.; Lee, J.; Liao, J.; Jiang, Z.; Zhang, L. A Novel Multidomain Polyketide Synthase is Essential for Zeamine Production and the Virulence of Dickeya zeae. Mol. Plant-Microbe Interact. 2011, 24, 1156-1164.

58. Masschelein, J.; Mattheus, W.; Gao, L.; Moons, P.; van Houdt, R.; Uytterhoeven, B.; Lamberigts, C.; Lescrinier, E.; Rozenski, J.; Herdewijn, P.; et al. A PKS/NRPS/FAS Hybrid Gene Cluster from Serratia plymuthica RVH1 Encoding the Biosynthesis of Three Broad Spectrum, ZeamineRelated Antibiotics. PLoS One 2013, 8, e54143.

59. Du, L.H.; Sanchez, C.; Shen, B. Hybrid Peptide-Polyketide Natural Products: Biosynthesis and Prospects Toward Engineering Novel Molecules. Metab. Eng. 2001, 3, 78-95.

60. Gaisser, S.; Hughes, C. A Locus Coding for Putative Non Ribosomal peptide/polyketide Synthase Functions is Mutated in a Swarming-Defective Proteus mirabilis Strain. Mol. Gen. Genet. 1997, 253, 415-427.

61. Nikaido, H. Antibiotic Resistance Caused by Gram-Negative Multidrug Efflux Pumps. Clin. Infect. Dis. 1998, 27, S32-S41.

62. Peng, W.; Nester, E. Characterization of a Putative RND-Type Efflux System in Agrobacterium tumefaciens. Gene 2001, 270, 245-252. 
63. Burse, A.; Weingart, H.; Ullrich, M. The Phytoalexin-Inducible Multidrug Efflux Pump AcrAB Contributes to Virulence in the Fire Blight Pathogen, Erwinia amylovora. Mol. Plant-Microbe Interact. 2004, 17, 43-54.

64. Valecillos, A.; Palenzuela, P.; Lopez-Solanilia, E. The Role of several Multidrug Resistance Systems in Erwinia chrysanthemi Pathogenesis. Mol. Plant-Microbe Interact. 2006, 19, 607-613.

65. Blumenthal, R.M.; Cheng, X. Restriction-Modification Systems. In Modern Microbial Genetics, 2nd ed.; Streips, U.N., Yasbin, R.E., Eds.; Wiley-Liss, Inc.: New York, NY, USA, 2002; ISBN: 978-0-471-38665-0, p. 178.

66. Pirhonen, Minna. University of Helsinki, Helsinki, Finland. Personal Communication, 2012.

67. Soballe, B.; Poole, R. Microbial Ubiquinones: Multiple Roles in Respiration, Gene Regulation and Oxidative Stress Management. Microbiology-(UK) 1999, 145, 1817-1830.

68. Brondijk, T.; Fiegen, D.; Richardson, D.; Cole, J. Roles of NapF, NapG and NapH, Subunits of the Escherichia coli periplasmic Nitrate Reductase, in Ubiquinol Oxidation. Mol. Microbiol. 2002, 44, 245-255.

69. Xie, F.; Cheng, G.; Xu, H.; Wang, Z.; Lei, L.; Li, Y. Identification of a Novel Gene for Biosynthesis of a Bacteroid-Specific Electron Carrier Menaquinone. PLoS One 2011, 6, e28995.

70. Juhas, M.; van der Meer, J.R.; Gaillard, M.; Harding, R.M.; Hood, D.W.; Crook, D.W. Genomic Islands: Tools of Bacterial Horizontal Gene Transfer and Evolution. FEMS Microbiol. Rev. 2009, 33, 376-393.

71. Schmidt, H.; Hensel, M. Pathogenicity Islands in Bacterial Pathogenesis. Clin. Microbiol. Rev. 2004, 17, 14-56.

(C) 2013 by the authors; licensee MDPI, Basel, Switzerland. This article is an open access article distributed under the terms and conditions of the Creative Commons Attribution license (http://creativecommons.org/licenses/by/3.0/). 